\title{
Sugars content in foods for infants and young children in Bulgarian markets
}

\section{Abstract}

Introduction: A sale of commercial foods for infants and young children in Bulgaria has grown rapidly in the last years. Recent evidence suggests that the nutritional content of some commercial foods for infants and young children may be associated with the risk factors for development of non-communicable diseases later on life.

The aims of the study were to collect data on available food products for infants and young children (0-36 months) in Bulgaria and to assess the sugars content in them. The survey is part of WHO Regional Office for Europe project: Commercial foods for infants and young children in the WHO European Region.

Materials and methods: The data was collected in November 2017 in two districts in capital city Sofia using the mobile questionnaire, developed by WHO Regional Office for Europe. Total sugars content of the products, where declared on the label, was recorded for 3153 products (breast-milk, follow-on formula, growing-up milk, complementary foods) from 91 shops (minimarket, pharmacy, drugstore, supermarket, baby goods store). Additional information was collected from the label for the presence of sugars or any other sweetening agents.

Results: he results reviled that the maximum total sugars content in different products ranged from $0 \mathrm{~g}$ per $100 \mathrm{kcal}$ to $25 \mathrm{~g}$ per 100 kcal. The products in Bulgarian markets have relatively high sugars contents and the energy from sugars was $15 \%$ in $67 \%$ of products; more than $30 \%$ in $49 \%$ of studded foods and more than $40 \%$ in $42 \%$ of foods for infants and young children. The most added sweetening agents were sugar $-17.7 \%$ and fruit juice concentrate $-16.9 \%$.

Discussion: hese products can promote preference for sweet foods an early age and increase the risk of overweight and dental caries. The very high levels of sugars present in commercial products in Bulgarian market are cause for concern.

\section{Conflict of Interest}

There is no conflict of interest. 